\title{
Preparing Nanoarchaeosome Containing Triptorelin Acetate and Evaluation of Its Cellular Toxic Effect on PC3 Prostate Cancer Cell Line
}

\author{
Zeynab Haj Mohammadi ${ }^{1}$, Shamsozoha Abolmaali ${ }^{2}$, Azim Akbarzadeh ${ }^{* 3}$ \\ 1. Faculty of Biotechnology Department for Microbial biotechnology, Semnan University, Semnan, Iran. \\ 2. Faculty of Biotechnology Department for Microbial biotechnology, Semnan University, Semnan, Iran. (0000-0001- \\ 6342-0828) \\ 3. Pasteur Institute of Iran, Tehran, Iran.
}

Article Type:

Original Article

\section{Article History:}

Received: 23 Apr. 2018

Revised: 12 Aug. 2018

Accepted:19 Aug. 2018

*Correspondence: Azim Akbarzadeh, Pasteur Institute of Iran, Tehran, Iran. azimakbarzadehkhya vi@yahoo.com

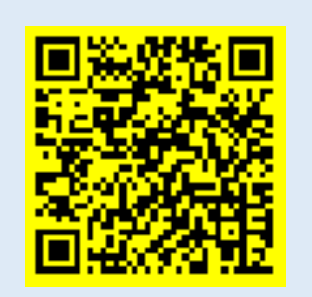

\begin{abstract}
Background and objectives: Prostate cancer is the second leading cause of mortality among men worldwide, and the eighth cancer-related cause of death in Iran. Male sex hormones (androgens) are the main cause of prostate cancer cells. Triptorelin is considered a synthetic decapeptide analogue of gonadotropin-releasing hormone ( $\mathrm{GnRH})$ in the treatment of prostate cancer. This study aimed to evaluate the methods to deal with the constraints in use of lipid nanoarchaeosomes. Due to their adjuvant feature and presence of ether links, archaeosomes are more stable in blood, compared to other lipidbased formulations. In the present study, the effects of nanoarchaeosomes containing triptorelin acetate on the cellular toxicity of the PC3 prostate cancer cell line were evaluated.

Methods: Halobacterium salinarum bacteria were cultured in HS medium, and their polar lipids of the membrane were removed applying the Blight\&Dye technique in order to obtain nanoarchaeosome containing membrane polar lipids. In the next stage, nanoarchaeosomes were prepared by the hydration of polar lipids. In addition, cell viability was evaluated using the MTT assay on the PC3 cell line.

Results: In this research, mean diameter of the drug-containing nanoarchaeosomes was estimated at $263 \mathrm{~nm}$ using the ZetaSizer device. Moreover, drug loading efficiency of nanoarchaeosomes was estimated at $98 \%$. Furthermore, the toxicity of the free and nanoarchaeosome drugs was assessed via the MTT assay, results of which were indicative of a mean of IC $500.22 \mu \mathrm{g} / \mathrm{ml}$ in this regard.

Conclusion: According to the results of the study, archaeosomes extracted from polar lipids were produced with high concentration and purity on the nanoscale. Considering the high stability, ease of production, level of drug loading, and toxic effect on cancer cells, nanoarchaeosomes can be used as a suitable technique for targeted drug delivery in future studies.
\end{abstract}

Keywords: Archaeosome, Nanotechnology, Triptorelin Acetate, Prostate Cancer

Copyright $($ 2018, Jorjani Biomedicine Journal has published this work as an open access article under the terms of the Creative Commons Attribution License (http://creativecommons.org/licenses/by-nc/4.0/) which permits noncommercial uses of the work while it is properly cited. 


\section{Introduction}

Prostate cancer is the most prevalent type of cancer in men and the second leading cause of mortality after lung cancer (1). About 200000 new cases are estimated annually, $20 \%$ of which lead to mortality of patients. In this regard, there is a greater incidence of latent prostate cancer. This increase in the prevalence of prostate cancer starts from $10 \%$ in the fifth decade of life and reaches $60 \%$ in the eighth decade. In the United States, the most commonly diagnosed cancer is prostate cancer, accounting for $30 \%$ of non-skin cancers in this country (2).

Surgery, chemotherapy, radiation therapy, immunotherapy, hormone therapy, and gene therapy are various therapeutic approaches to treat cancers, all of which are associated with therapeutic complications (3). Surgery is the first treatment of choice for numerous types of cancer. While surgery is effective in the early stages of cancer, the cases with a spread of cancer in the body require a concomitant use of surgery, chemotherapy, and radiation therapy. Radiation therapy changes the DNA of cancer cells and prevents the production of cancer cells by their degradation. Depending on the amount of radiation, the location of radiation irradiation, and the reaction of the body, conditions such as drought, skin burn, itching, hair loss, loss of appetite and energy, constipation or diarrhea, nausea, vomiting and disturbances may occur (4).

Chemotherapy is a unique therapeutic approach due to its flow in the whole body and access to cancer cells. Nevertheless, the toxic effects of chemotherapy drugs, the lack of selectivity of drugs against cancer cells, and their nonspecific performance along with complications such as energy and hair loss, nausea, vomiting, and increased risk of infection, are among the problems caused by this type of treatment. Men's sex hormones (androgens) are the most common cause of the majority of prostate cancer cells. Therefore, today, treatments based on the reduction of serum levels of androgens using the analogues of gonadotropinreleasing hormones $(\mathrm{GnRH})$ and antiandrogens are considered.

Among the important analogues with a long-term effectiveness, we can refer to buserelin, leuprorelin, nafarelin, goserelin and triptorelin. Generally, triptorelin is a synthetic decapeptide analogue of $\mathrm{GnRH}$ used in treatment of prostate cancer (5). Some of the complications of triptorelin acetate include nausea, stomachache, headache, dizziness, flushing, reduced sexual desire, palpitations, increased sweating, weakness, anger, acne, dry skin, backache, muscle and joints pain, sleep disorders, and paresthesia (6).

In addition, daily subcutaneous administration of $\mathrm{GnRH}$-analogue triptorelin acetate results in lack of cooperation of patients and an improper therapeutic response. Other defects in using GnRH analogues include inadequate drug concentration (due to low absorption), rapid drug withdrawal (due to peptide nature), unwanted distribution of medication to other tissues, toxic effect on healthy tissues, high solubility in water and instability of these analogues in body fluids and fluctuations in plasma levels of the drug, small index of some medications, and resistance to treatment by some cancer cells (7).

To solve these problems, lipid-based medication creating formulations based on nanotechnology were considered due to simple production method, creation of equivalent blood levels, production by polymers with high biocompatibility, ability to simultaneously transfer hydrophilic and 
lipophilic drugs, rapid blood purification, and controlled and concentrated release of active drug substance (7). The effects of nanotechnology on drug delivery have increased the impact of available therapies and formation of completely new therapies. Among the new therapies of drug delivery systems are pharmaceutical-polymeric drug combinations, polymer micelles, nanoparticles, liposomes and microparticles, which not only lead to the transfer of higher concentrations of the drug, but also result in targeting specific cells and organs. Therefore, reducing the side effects of the drug decreases its spread to non-target tissues (8).

While the use of each of the mentioned carriers is based on the type of medication and target tissue or cell, about 20 nanotechnology-based therapeutic products have been approved by the US Food and Drug Administration (FDA) for clinical applications to date and more of these products are undergoing clinical trials (9). Among the current formulations of liposomes in the world's drug market, we can refer to Doxil, DaunoXome, DepoCyt, and ONCO-TCS, which have loaded the medications of Doxorubicin, Daunorubicin, Cytarabine, and Vincristine, respectively (10). Despite the several advantages of liposomes, instability is considered as one of the main problems associated with their therapeutic use.

Archaeosome is lipid vesicles made from a collection of polar lipids extracted from membranes of archaebacteria. Extensive research has been conducted over the past decade to determine the potential applications of archaeosomes as vesicles of medication and vaccine delivery on a nanoscale (11). Archaebacteria have some advantages, compared to commonly used phospholipids to prepare liposomes. Among the most important advantages of polar bacterial phospholipids include the unique chemical structure of their plasma membrane lipids with higher stability of ether bonds, compared to ester linkages in a wide $\mathrm{pH}$ range, reduced crystalline formation and permeability of the membrane due to a branched structure, presence of saturated alkyl chains, which leads to high stability, compared to oxidative damage, and abnormal spatial and stereochemistry state of the main glycerol skeleton (8).

With this background in mind, this study aimed to evaluate the toxicity of archaeosomes containing triptorelin acetate on PC3 prostate cancer cell line.

\section{Materials and Methods}

\section{Compounds and Equipment}

Triptorelin acetate and (3- (4, 5Dimethylthiazol-2-yl)-2, 5-diphenyltetrazolium bromid $(\mathrm{mg} / \mathrm{ml})$ were purchased from Tofigh Darou and Sigma companies, respectively. On the other hand, ethanol, isopropanol, chloroform, dimethyl sulfoxide, casamino acids, yeast, extract, $\mathrm{KCL}, \mathrm{NaCl}, \mathrm{MnCl} 2 \cdot 4 \mathrm{H} 2 \mathrm{O}$, $\mathrm{MgSO}_{4} \cdot 7 \mathrm{H}_{2} \mathrm{O}$, and $\mathrm{FesO}_{4} .7 \mathrm{H}_{2} \mathrm{O}$ were bought from Merck Company.

The RPMI1640 culture was purchased from an Inogen distributor while the Halobacterium Salinarum (HS) strain with the IBRC-M No: 10715 (GCM: 9121, CCM: 2084) was bought from the Iranian Biological Resource Center. The PC3 prostatic cancer cell line was prepared by the cell bank of the Pasteur Institute of Iran. 


\section{Lipid Extraction}

The specific culture medium of HS containing $250 \mathrm{~g} / 1 \mathrm{NACL}, 20 \mathrm{~g} / 1 \mathrm{MgSO}_{4}, 3.64$ $\mathrm{g} / 1$ trisodium citrate, $2 \mathrm{~g} / \mathrm{l} \mathrm{KCL}$, and $10 \mathrm{~g} / \mathrm{l}$ peptone was prepared. The HS bacteria was cultured at $\mathrm{pH}=7.2$ and inoculated at $37^{\circ} \mathrm{C}$ and $170 \mathrm{rpm}$ for three days (12). After the growth of bacteria, the culture was centrifuged at 4000 rpm for 15 minutes. Following that, all polar lipids were extracted from HS via the Blight\&Dyer technique using a combination of methanol, chloroform, and water. In the next stage, archaeosome was prepared due to the hydration of polar lipids (12-16).

\section{Thin-layer Chromatography of Extracted Lipid}

The chromatography separation solution with ratios of $(85 / 22,5 / 10 / 4) \mathrm{ml}$ was prepared from the solutions of chloroform/methanol/acetic acid/water in the chromatography tank. The chromatography sheet loaded with $250 \mu$ of the polar lipids extracted from the solution in chloroform was placed inside the tank. After passing the separating solution from it, the chromatography sheet was removed from the tank, and stains appeared on the sheet by spraying the ammonium molybdate reagent solution and indirect heat (14).

\section{Nanoarchaeosome Preparation}

In order to prepare archaeosome containing triptorelin acetate, we added hydrated medium containing $\mathrm{mg}$ of triptorelin acetate into the thin layer of lipid film, which was a homogeneous mixture of $5.42 \mathrm{mg}$ cholesterol and 12.6 polar lipids of archaebacterial. Following that, sonication was performed for 10 minutes with a frequency of $60 \mathrm{~Hz}$ (Bandelin Sonorex Digitec) in order to harmonize the archaeosomes and preparation of a formulation with a better quality (17).
Determining the Size and Surface Charge of Nanoarchaeosome

The diameter of the particles was measured after preparing the archaeosome and drug loading. To this end, the drug sample was archaeosomized at first, and a 1:100 dilution ratio of non-drug archaeosome was prepared applying the physiological serum (phosphate buffer). Afterwards, its absorption at the wavelength of $630 \mathrm{~nm}$ was calculated. In the next stage, the mean diameter of archaeosomes was determined at the same dilution ratio (1:100) using the ZetaSizer Device (DLS) from Malvern Co., England (model: Nano ZS) with the range of six $\mu$ m-six $\mathrm{nm}$.

\section{Evaluation of Level of Drug Loading in Archaeosome}

In order to estimate the percentage of drug loading, we first prepared the drug's concentrations of $0.1,1,2,4$, and $6 \mathrm{mg}$ and measured their absorption at the wavelength of $220 \mathrm{~nm}$. Following that, the standard curve was drawn and its equation was determined. Then, a two-ml volume of each sample (drugcontaining archaeosome and non-drug archaeosome) was centrifuged in separate tubes for 60 minutes at $4{ }^{\circ} \mathrm{C}$ with $2000 \mathrm{rpm}$. In the next stage, the supernatant was collected and its absorption level was compared to the control sample (distilled water) at the wavelength of $220 \mathrm{~nm}$. In addition, the level of free drug was estimated using the standard diagram, followed by the estimation of the drug loading level applying the equation below.

Drug loading efficiency $=\frac{\text { The amoumt of drug trapped }}{\text { Total drug amount }} \times 100$

\section{Culture of PC3 Prostate Cancer Cell Line}

In this part of the research, $100 \mathrm{unit} / \mathrm{ml}$ PC3 prostate cancer cell line related to adenocarcinoma prostate with a human origin was cultured in RPMI1640, FBS10\%, Lglutamine, and penicillin and was incubated at 
$37^{\circ} \mathrm{C}$ with $5 \% \mathrm{CO} 2$ gas and 0.095 humidity. Cancer cells were treated with two types of transfosomal formulation with drug and control and free drug solution with descending concentrations of $100 \mu \mathrm{l}$ and evaluated after 24-72 hours of incubation. In addition, the non-treated cells were used as cells of the control group.

\section{Evaluation of Cellular Toxicity with MTT Assay}

A total of $10^{4}$ human cancer cells were placed in a 96-well plate as soluble, and the cells were allowed to be replaced and grow 24 hours inside the incubator in a new culture medium. After the adhesion of the cells to the bottom, $100 \mu \mathrm{l}$ of two formulations with drug and control and the free-drug soluble with descending concentrations of $(5.2,2.6,1.3$, $0.65,0.325,0.162 \mathrm{mg} / \mathrm{ml}$ ) were added to each well. Afterwards, the samples were placed in $\mathrm{CO} 2$ incubator for 48 hours at $37^{\circ} \mathrm{C}$. Following that, $100 \mu \mathrm{l}$ of the MTT solution was added at a final concentration of five $\mathrm{mg} / \mathrm{ml}$. Inoculation continued another three hours until the turning of MTT into formazan crystal in metabolically -active cells. After the removing of the plate from the incubator, the MTT solution was collected from the wells by a sampler, followed by adding $100 \mu \mathrm{l}$ of the isopropanol to each well. The presence of purple color after 15 minutes was recorded by Elisa Plate Reader at $570 \mathrm{~nm}$ wavelength. The results were expressed as the cytotoxicity percentage of the treated cells versus nontreated cells (control), which contributed to determining the level of IC 50 formulation using PHARM software.

\section{Results}

\section{Analysis of Extraction of Hallobacterium Polar Lipids}

In this section, we used thin-layer chromatography to confirm the presence of soluble polar lipids extracted from archaebacteria HS. Ammonium molybdate reagent solution, which detects polar lipids, confirmed the presence of polar lipids extracted with $0.875 \mathrm{RF}$ according to Figure 1 using the chloroform solvents, methanol, acetic acid, and water with ratios of $(85 / 22.5 / 10 / 4)$. In this test, lecithin was applied as standard.

Stain of polar lipids

Figure 1. Thin-layer Chromatography of HS Polar Lipids

\section{Nanoarchaeosome Characteristics}

\section{Size and Morphology of Nanoarchaeosome}

After the evaluation of size of drugcontaining archaeosomes and controls with the ZetaSizer device, the distribution percentage of size of nanoarchaeosome containing triptorelin acetate $(263 \mathrm{~nm})$ with PDI (polydispersity index) was reported at 0.363 , whereas the distribution percentage of the size of control nanoarchaeosome (no drugs) $(250 \mathrm{~nm})$ with PDI was recorded at 0.345 (Figure 2[a,b]). 


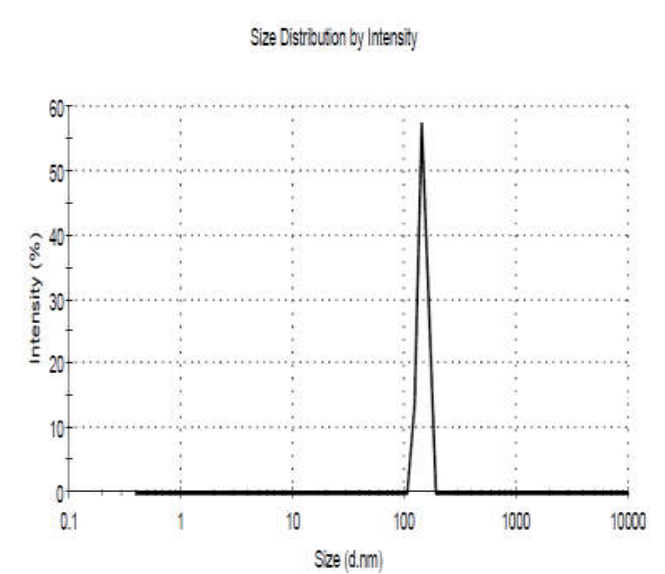

Diagram a) Size distribution curve of nanoarchaeosome containing triptorelin acetate

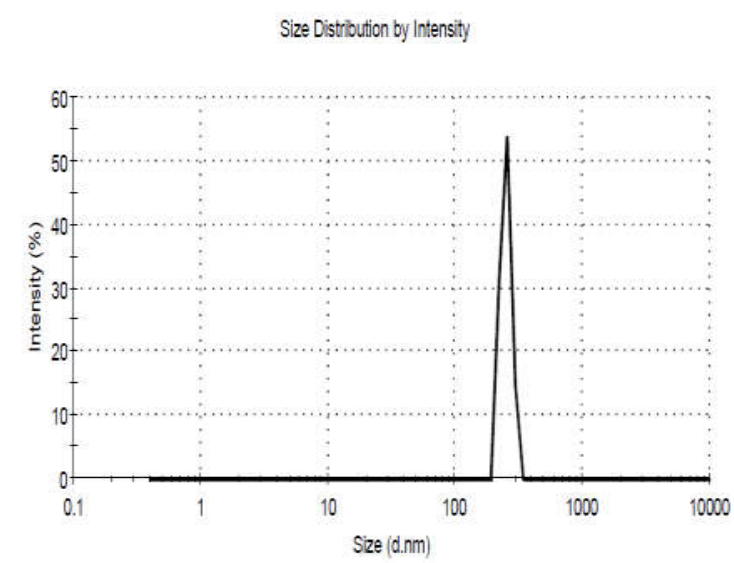

Diagram b) Size Distribution Curve of Control Nanoarchaeosome

Figure 2. Distribution Frequency of Particle Size of Drug-containing and Non-drug Nanoarchaeosomes
In addition, the surface charge of drugcontaining and control nanoarchaeosomes was measured by the ZetaSizer device and compared, results of which are presented in Table 3. In this regard, the surface charges of the control and drug-containing nanoarchaeosomes were obtained at -7.14 mvol and -15.4 mvol, respectively, where there was a two-fold increase in the load charge of the loaded nanoparticle, compared to the non-loaded nanoparticle.

\section{A) Surface Charge of Control Nanoarchaeosome Particles}

\begin{tabular}{|c|c|c|c|c|c|}
\hline \multicolumn{6}{|l|}{ Results } \\
\hline & & & Mean (mV) & Area (\%) & Width (mV) \\
\hline Zeta Potential $(\mathrm{mV})$ : & -7.14 & Peak 1: & 0.00 & 0.0 & 0.00 \\
\hline Zeta Deviation $(\mathrm{mV})$ : & 0.00 & Peak 2: & 0.00 & 0.0 & 0.00 \\
\hline Conductivity $(\mathrm{ms} / \mathrm{cm})$ : & 15.0 & Peak 3: & 0.00 & 0.0 & 0.00 \\
\hline
\end{tabular}

B) Surface Charge of Nanoarchaeosome Particles Containing Triptorelin Acetate

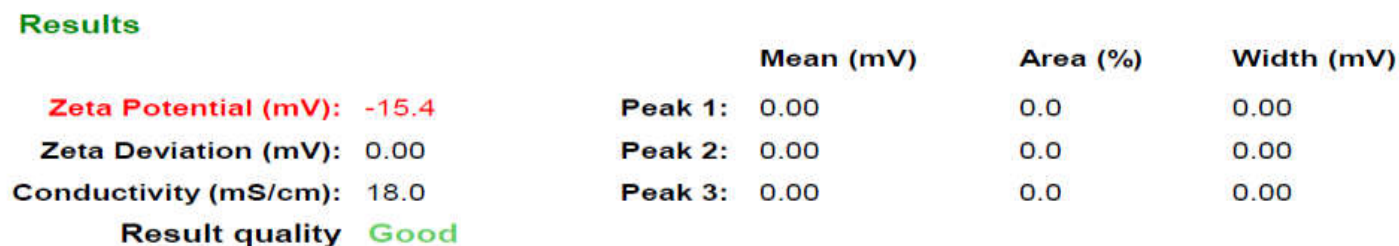



Increased potential of Zeta (surface charge)

and size of nanoarchaeosome after

formulation with triptorelin acetate was

indicative of drug loading in

nanoarchaeosome.
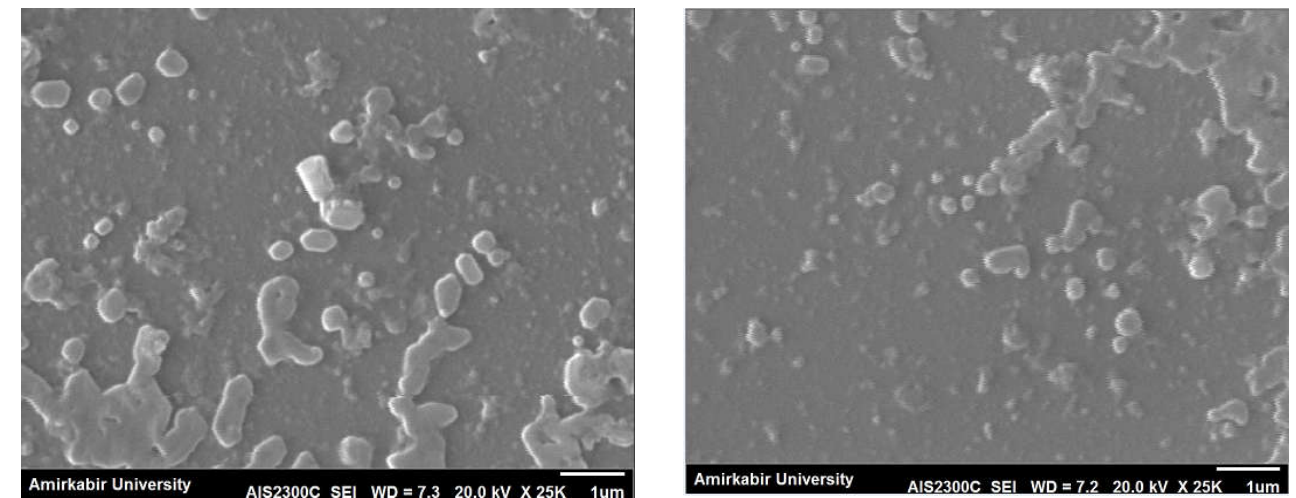

a) Morphology of Drug-containing Nanoarchaeosome

b) Morphology of Control Nanoarchaeosome

Figure 4. Morphology of Control and Drug-loaded Archaeosome Nanoparticles with Transmission Electron Microscopy (TEM)

\section{Loading Level}

The concentration of the released drug was estimated by optical absorption method, and its mean absorption level was estimated. By placing this variable on the linear equation, the concentration of the released drug was estimated at $0.12 \mathrm{mg}$. With the difference in initial drug value, the efficiency of the trapped drug was reported at 98.46.

\section{Evaluation of Toxicity and Lethality}

The toxicity level of nanoarchaeosome drug was assessed by MTT at various concentrations, and the level of $0.222 \mu \mathrm{g} / \mathrm{ml}$ was obtained according to mean 50 IC.

\section{Discussion}

In the present research, we evaluated the effect of drug-containing nanoarchaeosome on human PC3 prostate cancer cell line. The nanoarchaeosome was prepared using the polar lipids of the HS membrane. The mean diameter of drug-containing nanoarchaeosome was estimated at $263 \mathrm{~nm}$, whereas the efficiency of drug loading inside the nanoarchaeosome was reported to be $98 \%$. In addition, the cellular life of the PC 3 cell line was assessed via MTT assay, which confirmed the lethal effect of drug-containing nanoarchaeosome on tumor cells.

Prostate cancer is known as one of the most important and prevalent problems and one of the preventable cancers in men (18). The most important cause of this disease is the production and effect of testosterone hormones. Therefore, suppression of androgens by $\mathrm{GnRH}$ decapeptide analogue has been recognized as a more effective treatment of choice, compared to open radical prostatectomy, internal radiation therapy, and cryotherapy (19). Recognized as one of the LHRH agonists, triptorelin has the ability to reduce the serum testosterone level. This 
peptide has a better therapeutic effect, compared to similar drugs (20).

In a previous study, Heyns et al. marked that there was a higher patient survival rate in the triptorelin group, compared to the leuprolide group. During a nine-month period of triptorelin administration, the survival rate of patients was reported at $97 \%$, whereas the survival rate of patients administered with leuprolide was estimated at $90.5 \%$. Moreover, triptorelin better maintained the reduction of testosterone level during nine months, compared to the other medication (20). In another study by Morgan et al. (2018) conducted on the response level of gonadotropin-releasing hormone receptor and base cell of tumor cells to the agonist in vivo and in vitro, the effects of activating the GnRH receptor in HEK293 transfection cell (HEK293 [SCL60]) with Human ovarian cancer cells of SKOV3 and EFO21 were compared to human hepatoblastoma HepG2 cells and B35 neuroblastoma cells in mice. In this regard, results showed the difference in the level of $\mathrm{GnPH}$ receptor and apoptotic devices inside cellular cells (21).

However, the complications of triptorelin, fracture due to skeletal metastases (22), hematological and cardiovascular diseases limit the use of this medication (23). One of the considerable methods to reduce these complications is correcting the distribution of the drug in the body and entrapping it in microscopic drug carriers. As potential drug carrier system, the biocompatible lipid and nano microparticles increase the absorption of drugs in selective tissues through permeability and preservation effect, control the pharmacokinetics and drug's histological distribution profile, and improve intracellular penetration as a therapeutic indicator (24). The major issues in using lipid-based nanoarchaeosomes as drug-delivery systems include instability, short half-life, quick drug release and high costs on a high production scale (25). Lipids of the archaebacterial membrane have a special chemical structure and features, which distinguish them from liposome phospholipids (26).

In this respect, Gonzalez Parede et al. showed that archaeosome was a more appropriate nanocarrier, compared to the normal liposome, due to its stability, entrapping efficiency and antioxidant activities (11). In addition, in the study by Gonzalez Parede et al. conducted in 2010 to compare the effectiveness of betamethasone and probiotics used on skin, the archaeosome drug had a higher effectiveness, compared to the liposome drug (27). In 2010, the potential of archaeosomes as oral drug carriers for peptide medications (insulin) in diabetic mice was evaluated by Lee et al., who concluded that the archaeosomes were more resistant against gastrointestinal material (stomach and intestines) and improved the stability of fluorescent-labeled antigen (28). Furthermore, in a research by Krishna et al. on mice, it was indicated that archaeosome had no toxic impact on the samples even in long-term (6).

In another study by Vakilzadeh et al., the pulmonary delivery of loaded triptorelin in Pluronic based on nanomicell was carried out in mice. Results of the loading efficiency were reported at $84.36 \%$ with nanomicell size of $87.35 \mathrm{~nm}$ and Zeta potential of -12.8. In addition, a significant difference was observed between the soluble drug and the drug entrapped in nanomicell regarding the blood testosterone level (29). According to the evidence created here, the archaeosome carrier was selected for triptorelin.

According to the results of the present study, the level of cellular death in the PC3 cell line was reported at $0.222 \mu \mathrm{g} / \mathrm{ml}$ in the presence of nanoarchaeosome with $98.43 \%$ loading of triptorelin acetate based on mean IC 50. In a study by Patel et al. (2010), the 
level of tacrolimus (FK-506) loading in liposome was estimated at $31-49 \%$ (30). In addition, Bahita et al. showed that the loading level of tamoxifen, which is a SERM and prescribed in the treatment of cancers responding to the hormone, was approximately $45 \%$ in archaeosome (31). Comparison of drug loading of triptorelin acetate in nanoarchaeosome formulated with $0.023 \%$ cholesterol was estimated at $0.098 \%$.

In addition, archaeosome has been used by Rethore et al. for gene and peptide transport. In this regard, it was expected in the present study to transfer peptide triptorelin acetate with an acceptable efficiency (32). In evaluations performed by Moghimipour et al. to assess the ability of archaeosomes as drug carriers using methylene blue as model drug and recognitions carried out by differential scanning calorimetry (DSC), the stain loading inside the archaeosome carrier was confirmed (33). Similar to these results, evaluation of the effect of the formulation of 6-Jin-Ng pelgular nanoarcheosome on the growth of MCF-7 breast cancer cells in the Pasteur Institute of Iran showed the toxic effect of nanoarchaeosome on cell line (34).

In another study by Sharifat Salmani et al., which was performed in 2014 to produce archaeosome from membrane polar lipids applying Methanobrevibacter smihii by performing the polar lipids and archaeosomes produced, the proper placement of antigen inside archaeosomes and purity of these liposomes were confirmed, which was similar to our findings regarding lipid extraction and evaluation of archaeosome production (35).

Moreover, in comparison of the diameter of the nanoparticles obtained from the archaeosome used as DNA carrier vaccine at Tarbiat Modares University, the archaeosome containing plasmid $279 \mathrm{~nm}$ and an unloaded archaeosome $185 \mathrm{~nm}$ were obtained, compared to the production of triptorelin acetate carrying nanoarchaeosome, where the mentioned archaeosomes were estimated at $250 \mathrm{~nm}$ and $263 \mathrm{~nm}$, respectively (36).

\section{Conclusion}

Therefore and according to the comparison of our findings with similar studies, archaeosomization of triptorelin acetate is suggested as an effective method to kill PC3 cells as a more efficient technique, compared to other hormonal methods.

\section{Acknowledgements}

Not applicable

\section{Declarations}

\section{Funding source(s)}

This article was extracted from a master's thesis in University of Kerman (Code: 2446932) and was carried out in the biotechnology pilot section of the Pasteur Institute of Iran with the support of the mentioned university. Hereby, we extend our gratitude to the University of Semnan and dear colleagues in the biotechnology pilot section of Pasteur Institute of Iran.

\section{Ethics approvals and consent to participate Not applicable}

\section{Conflict of interest}

We declare that we have no financial or non-financial conflicts of interest related to the subject matter or materials discussed in the article. 


\section{References}

1. Jemal, A., et al., Cancer statistics, 2008. CA: a cancer journal for clinicians, 2008. 58(2): 71-96.

2. Machtay, M., et al., Higher biologically effective dose of radiotherapy is associated with improved outcomes for locally advanced nonsmall cell lung carcinoma treated with chemoradiation: an analysis of the Radiation Therapy Oncology Group. International Journal of Radiation Oncology* Biology* Physics, 2012. 82(1): 425-434.

3. Pollack, J.R., et al., Genome-wide analysis of DNA copy-number changes using cDNA microarrays. Nature genetics, 1999. 23(1): 41.

4. Jin, G.-H., et al., A comparative dosimetric study for treating left-sided breast cancer for small breast size using five different radiotherapy techniques: conventional tangential field, filed-infiled, tangential-IMRT, multi-beam IMRT and VMAT. Radiation Oncology, 2013. 8(1): 89.

5. Waxman J. Gonadotrophin hormone releasing analogues open new doors in cancer treatment. British medical journal (Clinical research ed.), 1987. 295(6606): 1084.

6. Krishnan, L., et al., Archaeosome vaccine adjuvants induce strong humoral, cell-mediated, and memory responses: comparison to conventional liposomes and alum. Infection and immunity, 2000. 68(1): 54-63.

7. Haley, B. and E. Frenkel. Nanoparticles for drug delivery in cancer treatment. In Urologic Oncology: Seminars and original investigations. 2008. Elsevier.

8. Choquet, C., et al., Stability of pressureextruded liposomes made from archaeobacterial ether lipids. Applied microbiology and biotechnology, 1994. 42(2-3): 375-384.

9. Shi, J., et al., Nanotechnology in drug delivery and tissue engineering: from discovery to applications. Nano letters, 2010. 10(9): 32233230 .

10. Hu, C.-M.J., S. Aryal, and L. Zhang, Nanoparticle-assisted combination therapies for effective cancer treatment. Therapeutic delivery, 2010. 1(2): 323-334.
11. González-Paredes, A., etal, Delivery systems for natural antioxidant compounds: Archaeosomes and archaeosomal hydrogels characterization and release study. International journal of pharmaceutics, 2011. 421(2): 321-331.

12. Oesterhelt, D. and W. Stoeckenius, [69] Isolation of the cell membrane of Halobacterium halobium and its fractionation into red and purple membrane, in Methods in enzymology. 1974, Elsevier. 667-678.

13. Dawson, K.S., K.H. Freeman, and J.L. Macalady, Molecular characterization of core lipids from halophilic archaea grown under different salinity conditions. Organic geochemistry, 2012. 48: 1-8.

14. Sprott, G.D., G.B. Patel, and L. Krishnan, Archaeobacterial ether lipid liposomes as vaccine adjuvants, in Methods in enzymology. 2003, Elsevier. 155-172.

15. Ferrante, G., J.C. Richards, and G.D. Sprott, Structures of polar lipids from the thermophilic, deep-sea archaeohacterium Methanococcus jannaschii. Biochemistry and Cell Biology, 1990. 68(1): 274-283.

16. Bligh, E.G. and W.J. Dyer, A rapid method of total lipid extraction and purification. Canadian journal of biochemistry and physiology, 1959. 37(8): 911-917.

17. Attar, A., et al., The potential of archaeosomes as carriers of pDNA into mammalian cells. Artificial cells, nanomedicine, and biotechnology, 2016. 44(2): 710-716.

18. Gao, X., et al., Diagnostic and prognostic markers for human prostate cancer. The prostate, 1997. 31(4): 264-281.

19. Touma, N.J., J.I. Izawa, and J.L. Chin, Current status of local salvage therapies following radiation failure for prostate cancer. The Journal of urology, 2005. 173(2): 373-379.

20. Heyns, C., et al., Comparative efficacy of triptorelin pamoate and leuprolide acetate in men with advanced prostate cancer. BJU international, 2003. 92(3): 226-231.

21. Morgan, K., et al., Gonadotropin-releasing hormone receptor levels and cell context affect tumor cell responses to agonist in vitro and in vivo. Cancer Research, 2008. 68(15): 6331-6340. 
22. ŠTĚPÁN, J.J., et al., Castrated men exhibit bone loss: effect of calcitonin treatment on biochemical indices of bone remodeling. The Journal of Clinical Endocrinology \& Metabolism, 1989. 69(3): 523-527.

23. Orwoll, E.S., et al., The rate of bone mineral loss in normal men and the effects of calcium and cholecalciferol supplementation. Ann Intern Med, 1990. 112(1): 29-34.

24. Peer, D., et al., Nanocarriers as an emerging platform for cancer therapy. Nature nanotechnology, 2007. 2(12): 751.

25. Grit, M. and D.J. Crommelin, Chemical stability of liposomes: implications for their physical stability. Chemistry and physics of lipids, 1993. 64(1-3): 3-18.

26. Koga, Y. and H. Morii, Recent advances in structural research on ether lipids from archaea including comparative and physiological aspects. Bioscience, biotechnology, and Biochemistry, 2005. 69(11): 2019-2034.

27. González-Paredes, A., et al., Archaeosomes as carriers for topical delivery of betamethasone dipropionate: in vitro skin permeation study. Journal of liposome research, 2010. 20(4): 269276.

28. Li, Z., et al., Investigation of archaeosomes as carriers for oral delivery of peptides. Biochemical and biophysical research communications, 2010. 394(2): 412-417.

29. Vakilzadeh, H., J. Varshosaz, and M. Minaiyan, Pulmonary Delivery of Triptorelin Loaded in Pluronic Based Nanomicelles in Rat Model. Current drug delivery, 2018. 15(5): 630640.

30. Patel, S., et al., Development and evaluation of liposomes for topical delivery of tacrolimus (Fk-506). Journal of Scientific Research, 2010. 2(3): 585 .
31. Bhatia, A., R. Kumar, and O.P. Katare, Tamoxifen in topical liposomes: development, characterization and in-vitro evaluation. J Pharm Pharm Sci, 2004. 7(2): 252-259.

32. Réthoré, G., et al., Archaeosomes based on synthetic tetraether-like lipids as novel versatile gene delivery systems. Chemical Communications, 2007(20): 2054-2056.

33. Moghimipour, E., et al., Archaeosome made from lipids extracted of Acidianus brierleyi as a new drug delivery system. Journal of Pure and Applied Microbiology, 2014. 8(2): 957-964.

34. Ahmadi, L., et al., to evaluate the effect of formulation of nano archaeosomal 6-gingerol on the growth of breast cancer MCF-7 cell line. 2015.

35. Sharifat Salmani, A., et al., Preparation of Archaeosome Adjuvant from membrane Polar lipids of large scale cultured Methanobreveibacter smithii. New Cellular and Molecular Biotechnology Journal, 2014. 4(15): 51-59.

36. Karimi, H., et al., Application of Archaeosome Nanoparticles as a DNA Vaccine Delivery System and Evaluation of its Effect in a C57BL/6 Tumor Model. Pathobiology Research, 2017. 19(4): 71-85.

\section{How to cite:}

Haj Mohammadi Z, Abolmaali Sh, Akbarzadeh A. Preparing Nanoarchaeosome Containing Triptorelin Acetate and Evaluation of Its Cellular Toxic Effect on PC3 Prostate Cancer Cell Line. Jorjani Biomedicine Journal. 2018; 6(2): 21-31. DOI: 10.29252/jorjanibiomedj.6.2.21 